\title{
Programmed death ligand-1 inhibitors potentially carry a lower risk of pneumonitis compared with programmed death-1 inhibitors in patients with non-small cell lung cancer
}

\author{
Minoru Fukuda ${ }^{1,2}$, Hiroyuki Yamaguchi ${ }^{2}$, Hiroshi Mukae $^{2}$, Kazuto Ashizawa $^{1,3}$ \\ ${ }^{1}$ Clinical Oncology Center, Nagasaki University Hospital, Nagasaki, Japan; ${ }^{2}$ Department of Respiratory Medicine, ${ }^{3}$ Department of Clinical \\ Oncology, Unit of Translational Medicine, Nagasaki University Graduate School of Biomedical Sciences, Nagasaki, Japan \\ Correspondence to: Minoru Fukuda, MD, PhD. Clinical Oncology Center, Nagasaki University Hospital, 1-7-1 Sakamoto, Nagasaki 852-8501, Japan. \\ Email: mifukuda258@nifty.com. \\ Provenance: This is an invited Editorial commissioned by the Executive Editor-in-Chief Jianxing He (Department of Cardiothoracic Surgery, The \\ First Affiliated Hospital of Guangzhou Medical University, Guangzhou, China). \\ Comment on: Pillai RN, Behera M, Owonikoko TK, et al. Comparison of the toxicity profile of PD-1 versus PD-L1 inhibitors in non-small cell lung \\ cancer: A systematic analysis of the literature. Cancer 2018;124:271-7.
}

Submitted Sep 14, 2018. Accepted for publication Sep 20, 2018.

doi: $10.21037 /$ jtd.2018.09.103

View this article at: http://dx.doi.org/10.21037/jtd.2018.09.103

Cancer cells are attacked by innate and acquired immune mechanisms; however, they can escape from immune surveillance via various mechanisms. Immunotherapies involving monoclonal antibodies against programmed death-1 (PD-1) and programmed death ligand-1 (PD-L1); i.e., checkpoint inhibitors, have been developed to inhibit the PD-1 pathway and cause T-cells to attack cancer cells. These treatments are used against various malignancies, including advanced non-small cell lung cancer (NSCLC) (1-4). The PD-1 inhibitors nivolumab and pembrolizumab and the PDL1 inhibitor atezolizumab have been demonstrated to be superior to docetaxel; i.e., they resulted in improved survival and lower toxicity, as a salvage treatment for advanced NSCLC $(1,2,4,5)$. The PD-L1 inhibitor durvalumab has been reported to be effective against stage III NSCLC when used after chemoradiotherapy (6). As these drugs are associated with serious and potentially fatal immune-related adverse events (irAE) (7-9), patients treated with immune checkpoint inhibitors must be monitored carefully. The recent development of checkpoint inhibitors has increased the number of treatment options for various types of cancer, and clinicians can have difficulty choosing the optimal agent.

Pillai et al. conducted a meta-analysis comparing the toxicities of the PD-1 and PD-L1 inhibitors used to treat advanced NSCLC (10). The analysis included 23 studies, 3,284 patients that were treated with $\mathrm{PD}-1$ inhibitors (nivolumab or pembrolizumab), and 2,460 patients that were treated with PD-L1 inhibitors (atezolizumab, durvalumab, or avelumab). There was no significant difference in the overall incidence of adverse events or the response rate between the groups. However, PDL1 inhibitor treatment resulted in a significantly lower incidence of pneumonitis (2\%) compared with PD-1 inhibitor treatment $(4 \%)(\mathrm{P}=0.01)$. The frequencies of irAE and hypothyroidism tended to decrease under PD-L1 inhibitor treatment $(\mathrm{P}=0.07)$. Although some of the trials of PD-L1 inhibitor treatment were carried out during an earlier phase, the abovementioned findings should help to guide future general practice and clinical trials to a certain extent.

The PD-1 pathway mainly contains two ligands, PDL1 and PD-L2. PD-L1 is constitutively present on both hematopoietic and non-hematopoietic cells and is regulated by external stimuli. In cancer cells, the PD-1/PD-L1 system inhibits the proliferation of T lymphocytes, cytokine release, and cytotoxicity, which leads to the exhaustion and apoptosis of tumor-specific $T$ cells and provides cancer cells with the opportunity to avoid immune responses (11). PD-L2 is a second PD-1 ligand. It is inducibly expressed on the surfaces of macrophages, dendritic cells, mast cells, and certain B cell populations (12,13). PD-L2 is suggested to regulate asthmatic responses through an interferon-gamma- 
dependent, but PD-1-independent, mechanism (14), and a lack of PD-L2 induces significant increases in transforming growth factor- $\beta$ and interleukin- $1 \alpha$ levels in the lungs, which can result in chronic airway hyperreactivity (15). PD-1 inhibitors can inhibit PD-L2-mediated immune reactions, whereas PD-L1 inhibitors do not. It is speculated that monoclonal antibodies against PD-L1 still allow PD-1 to interact with PD-L2, which results in lower rates of pneumonitis being observed during PD-L1 inhibitor treatment than during PD-1 inhibitor treatment.

Recently, the PD-L1 inhibitor durvalumab was introduced as a post-chemoradiotherapy treatment for stage III NSCLC (6), and further PD-1 inhibitors are also in development $(16,17)$. If PD-L1 inhibitors carry a lower risk of pneumonitis, they could become more important for combination with radiotherapy as a treatment for stage III NSCLC. In a study about the PD-L1 inhibitor durvalumab, the cases of 475 patients were evaluated, and 16 patients (3.4\%) experienced grade $3 / 4$ pneumonitis or radiation pneumonitis (6). In another study, the PD-1 inhibitor pembrolizumab was evaluated in 93 patients, and 5 patients (5.4\%) experienced grade $3 / 4$ pneumonitis (17). Thus, it seems that the PD-L1 inhibitor carried a slightly lower risk of pneumonitis; however, the latter study was not a full publication, and there is little data available about this issue.

PD-L1 inhibitors potentially carry a lower risk of pneumonitis and have advantage for clinical use compared with PD-1 inhibitors in various situation.

\section{Acknowledgements}

None.

\section{Footnote}

Conflicts of Interest: MF, HY, HM, and KA received honoraria from Ono pharmaceutical, MSD K.K. and Chugai Pharmaceutical. MF and HY received research funding from AstraZeneca.

\section{References}

1. Brahmer J, Reckamp KL, Baas P, et al. Nivolumab versus docetaxel in advanced squamous-cell non-small-cell lung cancer. N Engl J Med 2015;373:123-35.

2. Borghaei H, Paz-Ares L, Horn L, et al. Nivolumab versus docetaxel in advanced nonsquamous non-small-cell lung cancer. N Engl J Med 2015;373:1627-39.
3. Garon EB, Rizvi NA, Hui R, et al. Pembrolizumab for the treatment of non-small-cell lung cancer. $\mathrm{N}$ Engl J Med 2015;372:2018-28.

4. Herbst RS, Baas P, Kim DW, et al. Pembrolizumab versus docetaxel for previously treated, PD-L1-positive, advanced non-small-cell lung cancer (KEYNOTE-010): A randomized controlled trial. Lancet 2016;387:1540-50.

5. Rittmeyer A, Barlesi F, Waterkamp D, et al. Atezolizumab versus docetaxel in patients with previously treated non-small-cell lung cancer (OAK): A phase 3, openlabel, multicenter randomized controlled trial. Lancet 2017;389:255-65.

6. Antonia SJ, Villegas A, Daniel D, et al. Durvalumab after chemoradiotherapy in stage III non-small-cell lung cancer. N Engl J Med 2017;377:1919-29.

7. Reck M, Rodriguez-Abreu D, Robinson AG, et al. Pembrolizumab versus chemotherapy for PD-L1positive non-small-cell lung cancer. $\mathrm{N}$ Engl J Med 2016;375:1823-33.

8. Gettinger SN, Hom L, Gandhi L, et al. Overall survival and long-term safety of nivolumab (anti-programmed death 1 antibody, BMS-936558, ONO-4538) in patients with previously treated advanced non-small-cell lung cancer. J Clin Oncol 2015;33:2004-12.

9. Metro G, Ricciuti B, Brambilla M, et al. The safety of nivolumab for the treatment of advanced non-small cell lung cancer. Expert Opin Drug Saf 2017;16:101-9.

10. Pillai RN, Behera M, Owonikoko TK, et al. Comparison of the toxicity profile of PD-1 versus PD-L1 inhibitors in non-small cell lung cancer: a systematic analysis of the literature. Cancer 2018;124:271-7.

11. Okazaki T, Honjo T. PD-1 and PD-1 ligands: from discovery to clinical application. Int Immunol 2007;19:813-24.

12. Dong H, Zhu G, Tamada K, et al. B7-H1, a third member of the B7 family, co-stimulates T-cell proliferation and interleukin-10 secretion. Nat Med 1999;5:1365-9.

13. Latchman Y, Wood CR, Chernova T, et al. PD-L2 is a second ligand for PD-1 and inhibits $\mathrm{T}$ cell activation. Nat Immunol 2001;2:261-8.

14. Matsumoto K, Inoue H, Nakano T, et al. B7-DC regulates asthmatic response by an IFN-gamma-dependent mechanism. J Immunol 2004;172:2530-41.

15. Kerzerho J, Maazi H, Speak AO, et al. Programmed cell death ligand 2 regulates TH9 differentiation and induction of chronic airway hyperreactivity. J Allergy Clin Immunol 2013;131:1048-57.

16. Gerber DE, Urbanic JJ, Langer C, et al. Treatment 
design and rationale for a randomized trial of cisplatin and etoposide plus thoracic radiotherapy followed by nivolumab or placebo for locally advanced non-small-cell lung cancer (RTOG3505). Clin Lung Cancer 2017;18:333-9.

17. Durm GA, Althouse SK, Sadiq AA, et al. Phase II

Cite this article as: Fukuda M, Yamaguchi H, Mukae H, Ashizawa K. Programmed death ligand-1 inhibitors potentially carry a lower risk of pneumonitis compared with programmed death-1 inhibitors in patients with non-small cell lung cancer. J Thorac Dis 2018;10(Suppl 33):S4082-S4084. doi: 10.21037/ jtd.2018.09.103 trial of concurrent chemoradiation with consolidation pembrolizumab in patients with unresectable stage III nonsmall cell lung cancer: Hoosier Cancer Research Network LUN 14-179. J Clin Oncol 2018;36:abstr 8500. 